\title{
Immersion Natural Circulation Evaporative Cooling Server Cluster
}

\author{
Jun Yan \\ State Grid Xinyuan Company LTD \\ Beijing, China \\ E-mail: yanjun2008_28@163.com
}

\author{
Zhenguo Li \\ Institute of Electrical Engineering \\ Chinese Academy of Sciences \\ Beijing, China \\ E-mail: lzguo@mail.iee.ac.cn
}

\begin{abstract}
The foundation and running of Supercomputers and huge date centers consume a large amount of energy, and the cooling system takes up a large proportion. The application of advanced and efficient cooling technology has become the bottleneck problem in the electronic equipment field, which has significant meaning for energy conservation and is crucial for stable and reliable operation of the equipment. This paper introduces a new cooling technology demonstration prototype Immersion Natural Circulation Evaporative Cooling Server Cluster, which has been successfully applied to interactive experience area in the headquarters of State Grid Xinyuan Company LTD in Beijing.
\end{abstract}

Keywords-evaporative cooling, computer cooling, natural circulation, immersion.

\section{INTRODUCTION}

With the expansion of the data center and the computing center scale, system stability, reliability, noise, especially the energy consumption problem become more and more important. The traditional cooling method encountered a bottleneck problem of the hitherto unknown.

In some large server clusters, supercomputers and other devices. The air cooling has exposed the impassable drawbacks. Because of Beijing's poor air quality and heavy dust in the air, it's not ideal to put IT equipment into a traditional air-conditional room. Under the air cooling circumstances one room building requirements for increased, and it is not ideal heat and dust deposition will result in reduced equipment life [1]. On the other hand, with the increase of heating power density of electronic components and most of the equipment failures are caused by the burning of the chip. The cooling system energy consumption accounts for the proportion of the total power consumption room is very large [2], thus the cooling method has become a bottleneck problem.

Compared with the traditional cooling technology, the evaporation cooling uses the high insulating and moderate boiling point coolant and relies on the latent heat of vaporization to make the heating components cool by absorbing the more heat. Thus the evaporative cooling technology is an advanced and efficient cooling technology for the future server and mainframe computer.

Immersion natural circulation evaporative cooling server cluster in this paper is a fully enclosed system. The coolant is directly contacted with the heating element of the server cluster, so the heat is taken away quickly. The cooling system is no need to add extra power so it has the feature of energy saving. Above all there are no special requirements for the external space environment. The Power Usage Effectiveness has become more common in the international power efficiency of the data center to measure indicators and the PUE is the ratio of all energy consumed by the data center to the energy consumed by the IT, therefore the PUE is equal to 1.0 of the immersion natural circulation evaporative cooling server cluster.

\section{EVAPORATIVE COOLING TECHNOLOGY}

Evaporative cooling technology transfer heat with the phrase change of medium of high insulation, low boiling point to achieve cooling of the heat generating components. The application of this cost-effective new cooling technology in the electrical and electronic information equipment is in a leading position at home and abroad, and Institute of Electrical Engineering has a completely independent intellectual property rights.

The advantages of the evaporative cooling technology are as follows:

- The heat transfer efficiency of the phase change is higher and the cooling effect is better,

- The temperature distribution is uniform so that there is no dead zone and the temperature rise is lower,

- The working pressure of the evaporative cooling system is lower, thus the possibility of the coolant leakage is greatly reduced,

- The coolant has a good insulating property, which has the ability to extinguish the arc and restrain the occurrence and expansion of the electrical accident,

- The cooling technology is higher reliability, which can be seen from the circulation mechanism, the coolant and the cooling system,

- The cooling system has lower energy consumption and good economical efficiency.

Besides on the computer, this cooling technique also used in the power generation equipment, drives, transformers, converters and other electrical equipment. One of the most influential applications is 840MVA hydro-generator of the Three Gorges Project. The two units have been put into commercial operation for more than three years. They are stable and reliable till now.

\section{MATERIAL COMPATIBILITY RESEARCH}

In addition to the physical and chemical stability, environmental characteristics, heat transfer performance, insulation performance of the cooling medium, whether 
physical and chemical reaction occur among the cooling medium and a variety of electronic materials is the key issue. For example, the cooling medium corrodes electronic components, or some substances may dissolve in the medium and destroy chemical or physical properties of the medium. It should be studied clearly, which is the premise for evaporative cooling technology applied in the field of computer.

To solve this issue, the author and his research team design a variety of experimental apparatus to conduct special study [3]. The experiment and media screening are carried out for nearly three years and the team explored a reasonable medium screening method for IT equipment. The study [3] introduces in details the design and results of experiment, which will be important technology support for the security of supercomputer centers or large data centers.

\section{IMMERSION NATURAL CIRCULATION EVAPORATIVE COOLING SERVER CLUSTER}

The immersion Server Cluster works as follows: the overall server soaked in a cooling medium; heat generated by electronic components on the server is transmitted directly to the cooling medium and then taken away by the boiling cooling medium. Vaporized coolant rises to the top of the condensing unit by buoyancy and then transfer heat to surrounding air by phase change of condensing. Through the adapter board on the right side, all the power and signal lines are sealed and transferred into the device.
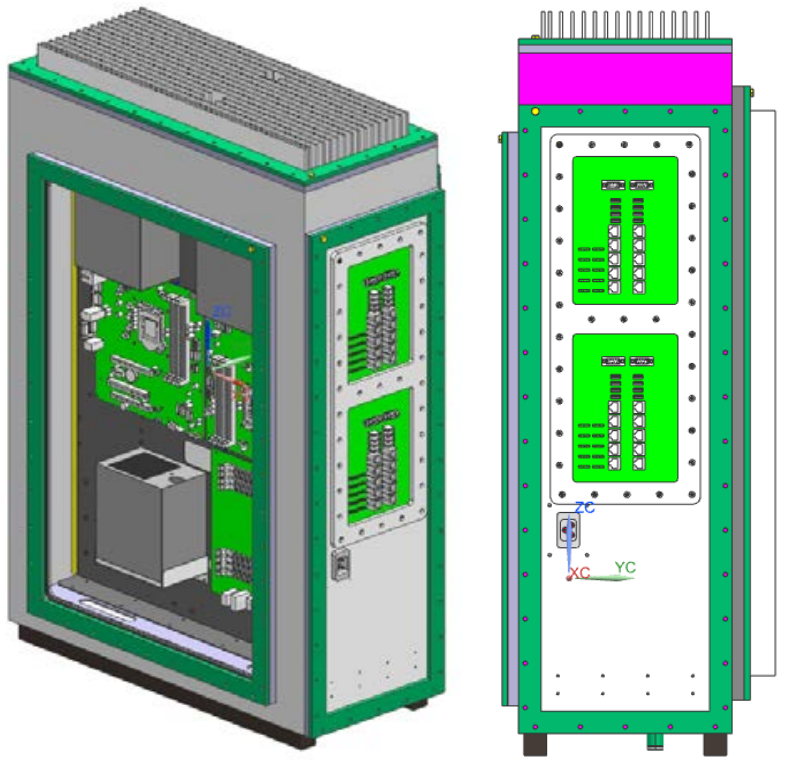

Figure 1. Immersion natural circulation evaporative cooling server cluster.

Without the high-speed fan in this cooling structure, the noise problem caused by the fan is avoided. Meanwhile, energy consumption of the system is reduced. In addition, due to the high thermal efficiency of evaporative cooling technology, computer devices run around 65 Rat tis less than their maximum allowable operating temperature and temperature distribution is even. So the reliability of the computing device can be greatly improved, service life can be greatly extended. The cooling can be widely used highend servers, personal computers and small-scale highperformance servers and supercomputer clusters.

Sealed box is placed in the external frame, as shown in the following figure2.

Exterior styling is to highlight the evaporative cooling technology and the technological sense. Inspired by "Transformers", it is designed by a professional industrial design company.
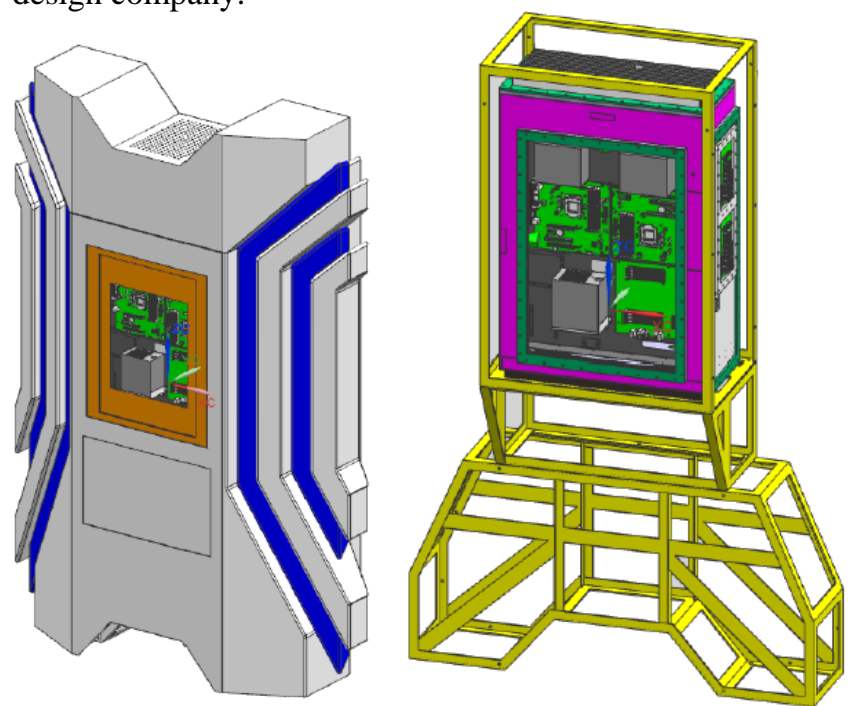

Figure 2. . Display cabinet of immersion server cluster.

\section{CONCLUSIONS}

This immersion server cluster has been successfully applied to interactive experience area in headquarters of State Grid Xinyuan Company in Beijing, which has been $7 * 24 \mathrm{~h}$ running for nearly two years without interruption, and provided data processing services to electronic sand table, fusion ring screen and other equipment.

As a general cooling technology, evaporative cooling technology is particularly suitable for highly integrated and high heat flux density large-scale electrical and electronic equipment. In view of the highly efficient cooling, energy saving, no noise and other advantages, the evaporative cooling technology will have a good application prospect. 

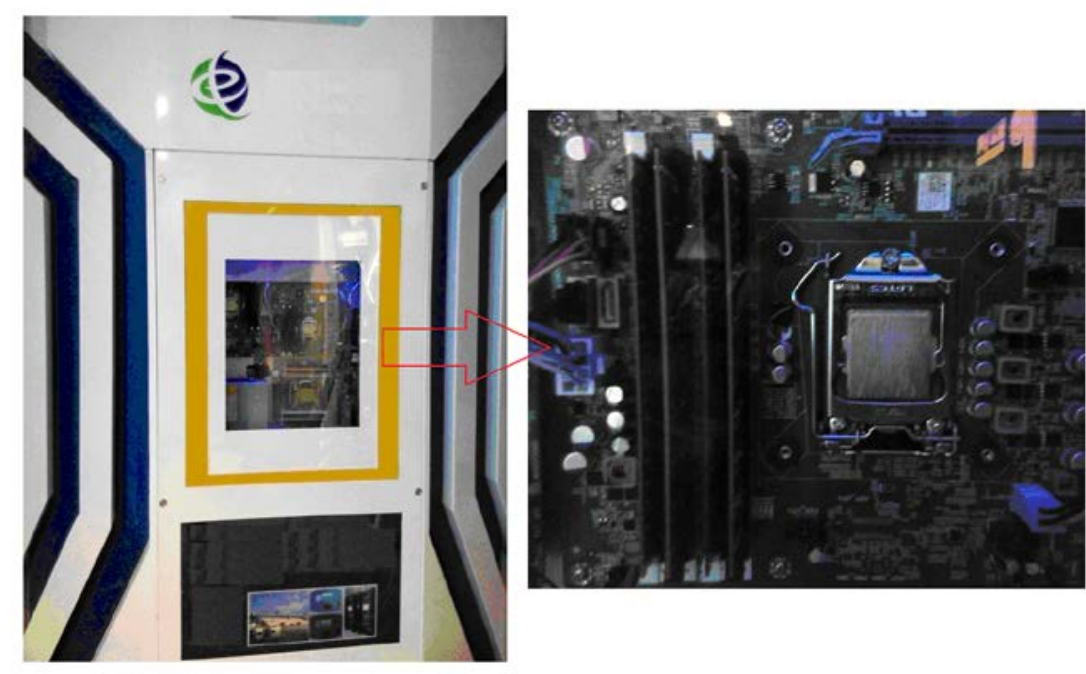

Figure3. Photos of Immersion Server Cluster

\section{REFERENCES}

[1] Lei Junxi etc, Research Progress on Chip Liquid cooling Technology. Science Technology and Engineering, 2008,8(15):4256-4263.

[2] Richard C. Chu. A review of IBM sponsored research and development projects for computer cooling[R]. Fifteenth IEEE SEMITHERM Symposium,1999:151-165.
[3] ZHANG Peng, RUAN Lin, XIONG Bin, Material compatibility research of evaporative cooling technology applied to large electronic equipment cooling, Advanced Technology of Electrical Engineering and Energy, Vol.33, NO.4 pp76-80, 2014.04. 